\title{
Genotype-guided treatment of oral P2Y 12 inhibitors: where do we stand?
}

\author{
Daniel MF Claassens ${ }^{1}$ \& Jurriën M ten Berg*,1,2 \\ ${ }^{1}$ Department of Cardiology, St Antonius Hospital, Nieuwegein, The Netherlands \\ 2Department of Cardiology, University Medical Centre Groningen, Groningen, The Netherlands \\ *Author for correspondence: jurtenberg@gmail.com
}

\begin{abstract}
"the POPular Genetics trial demonstrated that a personalized approach using genetic testing to de-escalate to clopidogrel benefits patient outcomes and is feasible in clinical practice in a high risk STEMI population"
\end{abstract}

First draft submitted: 6 November 2019; Accepted for publication: 6 November 2019; Published online: 20 January 2020

Keywords: acute coronary syndrome • clopidogrel • CYP2C19 • genotype-guided • myocardial infarction • P2Y12 inhibitor • prasugrel • ticagrelor

Dual antiplatelet therapy, consisting of aspirin and a $\mathrm{P}_{2} \mathrm{Y}_{12}$ inhibitor, remains the cornerstone of treatment in preventing recurrent thrombotic events such as stent thrombosis or spontaneous myocardial infarction (MI) after percutaneous coronary intervention (PCI) or acute coronary syndrome (ACS). In patients with ACS, the guidelines favor the stronger $\mathrm{P}_{2} \mathrm{Y}_{12}$ inhibitors ticagrelor or prasugrel over weaker clopidogrel $[1,2]$. This recommendation is based on the Platelet Inhibition and Patient Outcomes (PLATO) trial which demonstrated ticagrelor was superior in reducing MI and death as compared with clopidogrel and on the Trial to Assess Improvement in Therapeutic Outcomes by Optimizing Platelet Inhibition with Prasugrel-Thrombolysis in Myocardial Infarction 38, which demonstrated prasugrel was superior in reducing MI as compared with clopidogrel [3,4]. However, the improved efficacy of these stronger platelet inhibitors is counterbalanced by a higher bleeding risk.

Clopidogrel is a prodrug, which is transformed into its active metabolite by hepatic cytrochome P450 enzymes. The active metabolite irreversibly inhibits the $\mathrm{P}_{2} \mathrm{Y}_{12}$ receptor on platelets, resulting in inhibition of platelet aggregation [5]. However, clopidogrel-treated patients demonstrate a substantial individual response variability [6]. The most important gene to cause reduced clopidogrel effectiveness is the CYP2C19 gene [7-9]. Carriers of CYP2C19 loss-of-function (LoF) alleles are at an increased risk of thrombotic events [10-12]. This has prompted the US FDA and the European Medicines Agency to add a boxed warning to the label, describing that clopidogrel might not be effective in poor metabolizers [13].

More than 30 gene alleles have been identified [14]. The CYP2C19* 1 is the most prevalent $(60-70 \%$ of the population) and is regarded as the allele causing normal activity. CYP2C19*2 - 8 are LoF alleles. Of these, the CYP2C19*2 (15-35\%) and, depending on the population, *3 (0.02-9\%) alleles are, by far, the most common LoF alleles [15]. The CYP2C19*17 is regarded as a gain-of-function allele by many. Based on these assumptions, patients are divided into four different phenotype categories (Table 1) [16]. However, Lewis et al. demonstrated that CYP2C19*17 is not independently associated with clopidogrel response [17]. Furthermore, data on the influence of the CYP2C19* 17 allele on clinical outcomes are inconsistent and its clinical relevance is therefore questionable [15].

Despite the better efficacy of the stronger $\mathrm{P}_{2} \mathrm{Y}_{12}$ inhibitors, clopidogrel remains the most utilized platelet inhibitor of the three [15]. The reduced risk of bleeding and reduced costs, especially in non-western countries, probably plays an important part in this. In addition to the introduction of the stronger $\mathrm{P}_{2} \mathrm{Y}_{12}$ inhibitors, the introduction of newer generation stents have caused a dramatic decrease in thrombotic events, which has shifted attention to the prevention of bleeding complications [18]. This has spurred on research that tries to combine the improved efficacy of the stronger P2 $\mathrm{Y}_{12}$ inhibitors in patients that are unable to metabolize clopidogrel with the reduced risk of bleeding in patients that react properly to clopidogrel [19-21]. This can be achieved in two ways, either by using proof from platelet function testing or from genetic testing. Furthermore, it is possible to either chose a strategy in 
Table 1. CYP2C19 phenotypes based on genotypes.

\begin{tabular}{|lll|}
\hline Phenotype & Genotypes & Examples of diplotypes \\
\hline (Ultra)rapid metabolizer ${ }^{\dagger}$ & Carriers of one or two gain-of-function alleles & $* 1 / * 17, * 17 / * 17$ \\
\hline Extensive metabolizer & Non-carrier of LoF alleles & $* 1 / * 1$ \\
\hline Intermediate metabolizer & Carriers of one LoF allele & $* 1 / * 2, * 1 / * 3$ \\
\hline Poor metabolizer & Carriers of two LoF alleles & $\begin{array}{l}* 2 / * 2, * 2 / * 3, * 3 / * 3 \text { or combinations of two other LoF } \\
\text { alleles }(* 2-* 8)\end{array}$ \\
\hline
\end{tabular}

$\dagger$ Data on the existence of a gain-of-function allele are conflicting.

LoF: Loss-of-function.

which these tests are used to escalate a strategy from clopidogrel to the stronger $\mathrm{P} 2 \mathrm{Y}_{12}$ inhibitors or a de-escalation strategy from the stonger $\mathrm{P}_{2} \mathrm{Y}_{12}$ inhibitors to clopidogrel [21].

\section{Feasibility}

There have been many observational studies and randomized trials investigating the use of platelet function testing. However, there has never been a randomized trial which demonstrated a benefit in using platelet function testing over standard treatment Still, the latest European Society of Cardiology guidelines on myocardial revascularization give a class IIB recommendation to use platelet function testing to guide de-escalation of $\mathrm{P} 2 \mathrm{Y}_{12}$ inhibitor therapy in ACS patients [22]. However, there are some distinct advantages of genotyping over platelet function testing when it comes to feasibility. First, a point-of-care system like the Spartan Cube (Spartan Bioscience, Ontario, Canada) only requires a buccal swab, which is patient friendly and easy to use and returns a result in one hour [19]. Second, genetic testing can be performed at any time, either prior to elective PCI or in the cath lab in ST-segment elevation MI patients since it does not require stable platelet reactivity measurements to provide a reliable result [23]. Third, compared with the strategy used in for instance the Testing Responsiveness to Platelet Inhibition on Chronic Antiplatelet Treatment for Acute Coronary Syndromes trial, there is no need to return to the hospital for measuring platelet reactivity. At last, only one switch of $\mathrm{P}_{2} \mathrm{Y}_{12}$ inhibitors is necessary instead of two when patients are unresponsive to clopidogrel and have to be switched back to the potent platelet inhibitors [21].

\section{Escalation using CYP2C19 genotype-guided treatment}

Though the concept of CYP2C19 genotype-guided treatment has been around for some time, larger randomized trials evaluating the concept have been missing until recently. Results of the Reassessment of Anti-Platelet Therapy Using an Individualized Strategy Based on Genetic Evaluation trial, which included 187 patients, were already published in 2012. It demonstrated that one week after initiation of antiplatelet therapy there were significantly less patients with high on-treatment platelet reactivity in the genotype-guided group than in the standard treatment group, in which all patients received clopidogrel [24]. Since then, multiple smaller and mostly single center trials have been performed [25]. A meta-analysis from Kheiri et al. showed that there was no significant difference in major adverse cardiac events and bleeding events between genotype-guided treatment and standard treatment [25]. They, however, found a significantly lower rate of $\mathrm{MI}$ in the genotype-guided group (Hazard ratio [HR] $=0.44 ; 95 \% \mathrm{CI}$ : $0.28-0.70)$. However, this meta-analysis only included 2371 patients and there was a lot of heterogeneity in study population and in the definitions used in the different trials.

The trials in the meta-analysis investigated escalation of antiplatelet therapy from clopidogrel to ticagrelor or prasugrel, though these platelet inhibitors were also prescribed in the standard treatment arm in a significant portion of the patients. Currently, there are two larger ongoing randomized trials also investigating escalation of antiplatelet therapy after PCI. In the Tailored Antiplatelet Therapy Following PCI (NCT 01742117), 5300 patients undergoing PCI for stable coronary artery disease and ACS are randomized to standard treatment with clopidogrel or to genetic testing of the CYP2C19*2, *3 and * 17 alleles. Carriers of the LoF alleles are treated with ticagrelor, while noncarriers receive clopidogrel. Results of this trial are expected in the first half of 2019. A second trial, conducted in Saudi Arabia (NCT 01823185), is expected to include between 1500 and 2000 patients with STEMI. Patients in the standard treatment arm receive clopidogrel, while in the genotype-guided arm the CYP2C19*2 allele is tested. Carriers receive ticagrelor or prasugrel while noncarriers receive clopidogrel. Based on the meta-analysis, there might be an advantage in using genotyping to escalate treatment, but we have to wait for the results of these larger trials before we can draw more definitive conclusions. 


\section{De-escalation using CYP2C19 genotype-guided treatment}

Though clopidogrel is still the most widely used $\mathrm{P}_{2} \mathrm{Y}_{12}$ inhibitor, the use of the potent $\mathrm{P} 2 \mathrm{Y}_{12}$ inhibitors is preferred in ACS patients [1,2]. Based on this assumption the recently published CYP2C19 Genotype-Guided Antiplatelet Therapy in ST-Segment Elevation Myocardial Infarction Patients - Patient Outcome after Primary PCI (POPular Genetics) trial was conducted. Patients in the standard treatment arm received ticagrelor or prasugrel, while in the genotype-guided arm the CYP2C19*2 and *3 alleles were assessed. Carriers were also treated with ticagrelor or prasugrel, while noncarriers received clopidogrel [19]. The trial included 2488 STEMI patients undergoing primary PCI with more than $90 \%$ of the patients in the standard treatment arm receiving ticagrelor or prasugrel and more than $60 \%$ of the patients in the genotype-guided arm receiving clopidogrel. It concluded that a genotype-guided strategy was noninferior for net clinical benefit consisting of all-cause death, recurrent MI, definite ST, stroke and PLATO major bleeding ( $\mathrm{HR}=0.87$; $95 \% \mathrm{CI}$ : $\left.0.62-1.21, \mathrm{P}_{\text {for noninferiority }}<0.001\right)$, while it was superior in reducing combined PLATO major and minor bleeding $(\mathrm{HR}=0.78 ; 95 \% \mathrm{CI}: 0.61-0.98 ; \mathrm{p}=0.04)$. This reduction was mainly driven by a reduction in minor bleedings $(\mathrm{HR}=0.72$; $95 \% \mathrm{CI}: 0.55-0.94)$, with no difference in major bleeding ( $\mathrm{HR}=0.97$; $95 \% \mathrm{CI}: 0.58-1.63)$.

\section{Conclusion}

Currently, the guidelines still recommend a one-size-fits-all approach using the more potent platelet inhibitors ticagrelor and prasugrel. However, the POPular Genetics trial demonstrated that a personalized approach using genetic testing to de-escalate to clopidogrel benefits patient outcomes and is feasible in clinical practice in a high risk STEMI population. In the near future, other trials will provide an answer to the question whether a strategy using genotyping to escalate antiplatelet therapy will also benefit patients.

\section{Financial \& competing interests disclosure}

ten Berg JM reports grants and personal fees from AstraZeneca, and personal fees from Daiichi Sankyo, Eli Lilly, The Medicines Company, Accumetrics, Boehringer-Ingelheim, Bayer, Bristol Meyers Squibb, Pfizer and Ferrer, outside the submitted work. Claassens DMF has no disclosures. The authors have no other relevant affiliations or financial involvement with any organization or entity with a financial interest in or financial conflict with the subject matter or materials discussed in the manuscript apart from those disclosed.

No writing assistance was utilized in the production of this manuscript.

\section{References}

1. Ibanez B, James S, Agewall S et al. 2017 ESC guidelines for the management of acute myocardial infarction in patients presenting with ST-segment elevation: the task force for the management of acute myocardial infarction in patients presenting with ST-segment elevation of the European Society of Cardiology. Eur. Heart J. 39, 119-177 (2018).

2. Levine GN, Bates ER, Bittl JA et al. 2016 ACC/AHA guideline focused update on duration of dual antiplatelet therapy in patients with coronary artery disease: a report of the American College of Cardiology/American Heart Association Task Force on Clinical Practice Guidelines. J. Am. Coll. Cardiol. 68, 1082-1115 (2016).

3. Wallentin L, Becker RC, Budaj A et al. Ticagrelor versus clopidogrel in patients with acute coronary syndromes. N. Engl. J. Med. 361, 1045-1057 (2009).

4. Wiviott SD, Braunwald E, McCabe $\mathrm{CH}$ et al. Prasugrel versus clopidogrel in patients with acute coronary syndromes. N. Engl. J. Med. 357, 2001-2015 (2007).

5. Van Werkum JW, Heestermans AA, Deneer VHM et al. Clopidogrel resistance: fact and fiction. Future Cardiol. 2, $215-228$ (2006).

6. Breet NJ, Van Werkum JW, Bouman HJ et al. Comparison of platelet function tests in predicting clinical outcome in patients undergoing coronary stent implantation. JAMA 303, 754-762 (2010).

7. Shuldiner AR, O'Connell JR, Bliden KP et al. Association of cytochrome P450 2C19 genotype with the antiplatelet effect and clinical efficacy of clopidogrel therapy. JAMA 302, 849-857 (2009).

8. Simon T, Verstuyft C, Mary-Krause M et al. Genetic determinants of response to clopidogrel and cardiovascular events. N. Engl. J. Med. 360, 363-375 (2009).

9. Mega JL, Simon T, Collet JP et al. Reduced-function CYP2C19 genotype and risk of adverse clinical outcomes among patients treated with clopidogrel predominantly for PCI: a meta-analysis. JAMA 304, 1821-1830 (2010).

10. Angiolilo DJ, Fernando-Ortiz A, Bernardo E et al. Variability in individual responsiveness to clopidogrel: clinical implications, management and future perspectives. J. Am. Coll. Cardiol. 49, 1505-1516 (2007).

11. Collet JP, Hulot JS, Pena A et al. Cytochrome P450 2C19 polymorphism in young patients treated with clopidogrel after myocardinal infarction: a cohort study. Lancet 373, 309-317 (2009). 
12. Harmsze AM, Van Werkum JW, Ten Berg JM et al. CYP2C19*2 and CYP2C19*3 alleles are associated with stent thrombosis: a case-control study. Eur. Heart J. 31, 3046-3053 (2010).

13. Holmes DR, Dehmer GJ, Kaul S, Leifer D, O'Gara PT, Stein CM. ACCF/AHA clopidogrel clinical alert: approaches to the FDA "boxed warning": a report of the American College of Cardiology foundation task force on clinical export consensus documents and the American Heart Association endorsed by the Society of Cardiovascular Angiography and Interventions and the Society of Thoracic Surgeons. J. Am. Coll. Cardiol. 56, 321-341 (2010).

14. Scott SA, Sangkuhl K, Shuldiner AR et al. PharmGKB summary: very important pharmacogene information for cytochrome P450, family 2, subfamily C, polypeptide 19. Pharmacogenet. Genomics 22, 159-165 (2012).

15. Moon JY, Franchi F, Rollini F et al. Role of genetic testing in patients undergoing percutaneous coronary intervention. Expert Rev. Clin. Pharmacol. 11, 151-164 (2018).

16. Scott SA, Sangkuhl K, Stein CM et al. Clinical pharmacogenetics implementation consortium guidelines for CYP2C19 genotype and clopidogrel therapy: 2013 update. Clin. Pharmacol. Ther. 94, 317-323 (2013).

17. Lewis JP, Stephens SH, Horenstein RB et al. The CYP2C19*17 variant is not independently associated with clopidogrel response. J. Thromb. Haemost. 11, 1640-1646 (2013).

18. Sibbing D, Aradi D, Alexopoulos $\mathrm{D}$ et al. Updated expert consensus statement on platelet function and genetic testing for guiding $\mathrm{P} 2 \mathrm{Y}_{12}$ receptor inhibitor treatment in percutaneous coronary intervention. JACC Cardiovasc. Interv. 12, 1521-1537 (2019).

19. Claassens DMF, Vos GJA, Bergmeijer TO et al. A genotype-guided strategy for oral P2Y $\mathrm{Y}_{12}$ inhibitors in primary PCI. N. Engl. J. Med. 381, 1621-1631 (2019).

20. Janssen PWA, Bergmeijer TO, Vos GA et al. Tailored P2 12 inhibitor treatment in patients undergoing non-urgent PCI-the POPular Risk Score Study. Eur. J. Clin. Pharmacol. 75, 1201-1210 (2019).

21. Sibbing D, Aradi D, Jacobshagen C et al. Guided de-escalation of antiplatelet treatment in patients with acute coronary syndrome undergoing percutaneous coronary intervention (TROPICAL-ACS): a randomised, open-label multicentre trial. Lancet 390, 1747-1757 (2017).

22. Neumann FJ, Sousa-Uva M, Ahlsson A et al. 2018 ESC/EACTS guidelines on myocardial revascularization. Eur. Heart J. 40, 87-165 (2019).

23. Bergmeijer TO, Vos GJA, Claassens DMF et al. Feasibility and implementation of CYP2C19 genotyping in patients using antiplatelet therapy. Pharmacogenomics 19, 621-628 (2018).

24. Roberts JD, Wells GA, Le May MR et al. Point-of-care genetic testing for personalisation of antiplatelet treatment (RAPID GENE): a prospective, randomised, proof-of-concept trial. Lancet 379, 1705-1711 (2012).

25. Kheiri B, Osman M, Abdalla A et al. CYP2C19 pharmacogenetics versus standard of care dosing for selecting antiplatelet therapy in patients with coronary artery disease: a meta-analysis of randomized clinical trials. Catheter. Cardiovasc. Interv. 93, 1246-1252 (2019). 